\title{
Immunodeficiency and autoimmunity during biological disease-modifying antirheumatic drug therapy
}

\author{
Anna Czekalska, Dominik Majewski, Mariusz Puszczewicz \\ Department of Rheumatology and Internal Medicine, Poznan University of Medical Sciences, Poland
}

\begin{abstract}
Biological disease-modifying antirheumatic drugs target specific components of the immune response related to pathogenesis of autoimmune and inflammatory diseases. Introduction of biologic therapies has enabled better disease control than conventional drugs and thus a reduction in comorbidity and mortality. However, there is concern about adverse effects of these drugs including infections, cancers and drug-induced autoimmune diseases. Patients undergoing biologic treatment are at small but significant risk of serious infections. The overall risk of malignancies in patients on biologics compared with the general population is not increased, but there is evidence of a higher risk of individual cancers. Surprisingly, biological treatment may induce autoantibody production and, rarely, development of autoimmune diseases. A growing body of literature has evaluated the risk of adverse effects during biologic therapies. This paper outlines adverse effects of biological disease-modifying antirheumatic drugs related to immune system disorders, both immunodeficiency and autoimmunity.
\end{abstract}

Key words: biologic agents, biological disease-modifying antirheumatic drugs, adverse effects.

\section{Introduction}

The treatment of rheumatic inflammatory diseases has been revolutionized by the development of biological therapies. Biological disease-modifying antirheumatic drugs (bDMARDs) are divided into groups based on their molecular target including tumor necrosis factor $\alpha$ inhibitors (TNFis: infliximab, etanercept, adalimumab, certolizumab pegol, golimumab), CD-20 activity blocker (rituximab), anti-interleukin-6-receptor antibodies (anti-IL-6R: tocilizumab, sarilumab), anti-interleukin 17/anti-interleukin-17-receptor antibodies (anti-IL-17/IL-17R: secukinumab, ixekizumab, brodalumab), anti-interleukin-1-receptor antibody (anti-IL-1R: anakinra), anti-B-lymphocyte stimulator antibody (anti-BLyS: belimumab) and T-cell costimulation inhibitor (abatacept).

Although the mechanism of action is different for each group, the bDMARDs' therapeutic effect is achieved by dampening the immune response and reducing inflammation. The therapeutic efficacy and safety of the above-mentioned drugs have been well established and the agents have been approved by the U.S. Food and Drug Administration [1].

However, bDMARDs have adverse effects (AEs) including the most common injection site reactions and rare but severe complications. There have been reports of an increased risk of serious infections and malignancies, due to immunosuppression, and paradoxically, an increased risk of autoimmune diseases. Specific risk with bDMARDs include heart failure with TNF inhibitors, depression with rituximab, neutropenia with anakinra, gastrointestinal perforations with IL-6R antagonists and inflammatory bowel disease with IL-17 antagonists.

Immunodeficiency occurs when one or more components of the immune system are compromised. Biological therapies target inflammatory and immune pathways and therefore may cause immunodeficiency. Since the main function of the immune system is defense against infection, immunosuppression may lead to serious infections defined as life-threatening or requiring hospitalization [2]. Moreover, the immune system plays a critical role 
in surveillance of cancers. Tumor-associated antigens are recognized by $T$ cells, which results in cell-mediated and humoral immune responses against cancer cells [3]. Immunocompromised individuals have an elevated risk of cancer compared with the general population [4]. Therefore, there was a concern that treatment with bDMARDs may increase the risk of malignancies.

Autoimmunity is an abnormal immune response directed against self-antigens, which results in tissue damage and chronic inflammation [5]. Common autoimmune diseases include rheumatoid arthritis (RA), systemic lupus erythematosus (SLE) and inflammatory bowel disease (IBD). Biological treatment dampens the inflammatory cascades and thus slows down the progression of these conditions. Surprisingly, bDMARD therapy may lead to induction of autoantibodies and development of drug-induced autoimmune diseases [6].

\section{Infections}

Due to their immunosuppressive effect, bDMARDs are associated with an increased risk of infections. Data from two large registries, the Anti-Rheumatic Therapy In Sweden (ARTIS) and the British Society for Rheumatology Biologics Registers (BSRBR), suggest that the risk of serious infections (SIs) in RA patients is small, but clinically significant [7, 8].

A meta-analysis conducted by Singh et al. [9] shows that the absolute risk of serious infections associated with biologic agents compared to that with conventional synthetic DMARDs (csDMARDs) is increased and dose-dependent: 6 per 1000 RA patients treated each year and 17 per 1000 for standard and high dose biological therapy, respectively.

One study comparing bDMARDs with the general population found that the standardized incidence rate of serious infections is 16-21 for TNFis [10]. Moreover, the risk of serious infections in RA patients on anti-TNF treatment is time-dependent and is significantly elevated early on during treatment $[7,11]$.

In general the risk across bDMARDs is similar $[12,13]$. However, several studies indicate infliximab and adalimumab as agents with a higher risk of infections than etanercept $[14,15]$.

The spectrum of infections during bDMARD therapy is broad. The etiology of the infections may be bacterial, viral, invasive fungal and parasitic. The most frequent infections in patients on biologic drugs are pneumonia, other respiratory infections and infections of bones and joints [16]. The infectious disease course during immunosuppressive therapy is frequently severe.

There is a particular concern among rheumatologists about the incidence of tuberculosis (TB) and the risk of latent TB reactivation in patients on biologic therapy. Bacterial lipopolysaccharides (LPS) are known to induce macrophage activation and secretion of large amounts of TNF [17], which allows the host to fight the infection. Mycobacterium tuberculosis enhances LPS-induced TNF production [18]. Importantly, TNF was shown to be essential in control of TB and cannot be replaced with other cytokines [19]. TNF-deficient mice are highly susceptible to infections including reactivation of latent TB [20].

It has been shown that RA patients not exposed to bDMARDs have a 4-fold increased risk of TB compared to the risk in the general population [21]. Corticosteroid treatment is an another risk factor for developing TB [22]. Moreover, host defense mechanisms that act to control TB infection are affected during anti-TNF therapy [23]. Thus, screening for TB should be conducted in patients starting and receiving biologic drugs. There are two test methods available: the purified protein derivative (PPD) test and the interferon-gamma release assays (IGRAs) including the QuantiFERON-TB Gold assay [24]. The PPD measures type IV hypersensitivity in response to antigens of Mycobacterium tuberculosis and has low sensitivity in immunosuppressed patients and is positive in patients vaccinated with the BCG vaccine. IGRAs test ex vivo the reactivity of patient-derived T cells, which is also dependent on the patient immune status. Furthermore, there is low to moderate agreement between the PPD and IGRAs [25]. In view of the limitations, screening with both tests is proposed. However, IGRAs are recommended if the patient has been vaccinated with the BCG vaccine [26].

It is well known that reactivation of herpes zoster, another opportunistic infection, may lead to spread of the disease and death in immunosuppressed individuals [27]. Analysis of large databases has shown that there is no increased risk of herpes zoster infection in RA patients on bDMARDs versus csDMARDs $[28,29]$. Stratified analysis of the randomized controlled trials' data performed by Marra et al. [29] demonstrated a greater risk of herpes zoster events for non-TNF agents compared to TNF inhibitors. However, this finding needs to be confirmed in further studies. It is noteworthy that the rate of herpes zoster infections in RA patients is reported to be more than double compared to the general population and clinical vigilance is needed [30].

There is also concern among clinicians about the risk of progressive multifocal leukoencephalopathy $(\mathrm{PML})$ in patients receiving biological therapy. $P M L$ is a demyelinating disease of the central nervous system caused by reactivation of latent JC polyomavirus. In rheumatology, the highest risk of PML is associated with rituximab treatment [31]. Nevertheless, a cumulative analysis of PML cases in patients with RA or vasculitis demonstrated that PML events are very rare and remained stable despite increasing use of rituximab [32].

Sepsis may be a complication in serious infection. Sepsis is a major concern in patients with serious in- 
fections because it ends in death in $30-50 \%$ of cases [33]. Interestingly, the effect of bDMARD therapy on the risk of sepsis following serious infections in RA patients seems to be favorable.

Richter et al. [16] conducted an observational cohort study and investigated outcomes of serious infection in a large group of patients $(n=947)$ recruited to the German biologics registry Rheumatoid Arthritis: Observation of Biologic Therapy (RABBIT). $11.7 \%$ of cases of serious infections developed into sepsis and 63\% of these had a fatal outcome. It is noteworthy that the risk of sepsis and mortality was significantly reduced in patients on bDMARDs compared with those on csDMARDs. Sepsis is linked to an overabundant inflammatory response. In the serum of patients with sepsis increased levels of TNF and IL-1 are detected [33]. Thus, attempts were made to treat the patients with antibodies against TNF and with IL1R antagonist. A systematic analysis of studies concerning anti-TNF therapy in sepsis suggested that the treatment significantly reduces mortality [34], which is consistent with the observation in an RA patient cohort.

Surprisingly, a placebo-controlled trial did not show a reduction in mortality in patients with sepsis treated with ILIR antagonist [35]. In this study, the treatment was administered after development of sepsis when the inflammation cascade had begun. The timing of inflammatory cytokine blockade may influence the effect of therapy [36]. Better outcomes of sepsis in patients during bDMARD treatment compared to cases when drugs were administered after sepsis development suggest that an early start of therapy is important. Further studies are necessary to confirm the hypothesis.

\section{Malignancies}

After the introduction of bDMARD therapy there was a growing interest in the risk of malignancies in patients during the treatment. Because TNF is an essential cytokine in defense against cancer, it was assumed that its blockade might facilitate the development of malignancies. An early meta-analysis indicated an over three-fold increase in cancers in patients treated with infliximab or adalimumab versus placebo [37] and fueled concerns about the risk of malignancies during anti-TNF therapy. However, further studies did not show an increase in the overall risk of cancers in patients on bDMARDs both in comparison to the general population and to patients on csDMARDs. In the group of patients from the Spanish Registry for Adverse Events of Biological Therapy in Rheumatic Diseases (the BIOBADASER), including 2531 cases of RA, 1488 cases of spondyloarthropathies and 675 cases of other rheumatic conditions, it was demonstrated that overall cancer rates in patients with rheu- matic diseases exposed to anti-TNF agents are no higher than in the background population [38]. Similarly, analyses based on data from two other large biologics registers - RABBIT and the BSRBR - showed no significant differences in the incidence of malignancies in patients on anti-TNF drugs and patients on CSDMARDs $[39,40]$.

Regarding the risk of individual cancers, RA patients on biologic agents, as compared with the general population, were indicated to have a higher risk of hematologic malignancies [41]. However, no increased risk of lymphoma was found in patients on TNFis compared with those on csDMARDs [42]. In RA the overall incidence of lymphomas is twofold higher in comparison to that in the normal population. The risk of lymphoma in RA patients seems to be more dependent on RA itself and disease activity than on the treatment [42], which is consistent with the knowledge that chronic inflammatory diseases increase the risk of malignancy.

A recent study based on data from the Swedish registry showed that women with RA on anti-TNF therapy are at increased risk of cervical cancer compared with biologics-naive women with RA [43]. The relative risk of invasive cervical cancer in the group on TNFi was doubled. Further analyses are needed to examine whether the increased risk of cervical cancer is causally linked with anti-TNF treatment.

The risk of malignancies in patients treated with bDMARDs other than TNFis is less investigated. In a recent prospective cohort study based on the ARTIS register database the incidence of malignant neoplasms in RA patients treated with abatacept, tocilizumab and rituximab was evaluated [44]. There were no statistically significant differences in the risk for solid and hematologic malignancies, excluding non-melanoma skin cancer, between patients on non-TNFis and patients on cSDMARDs or the general population. An increased risk of non-melanoma skin cancer, compared with csDMARDs, was found for abatacept. This is in good agreement with analysis of long-term safety of abatacept, which indicated non-melanoma skin cancer as the most frequent malignancy in abatacept users [45].

Biologic treatment may theoretically interfere with immune cancer surveillance and clinical guidelines warn against the use of anti-TNF agents in patients with a recent history of cancers [26]. To investigate the risk of recurrence of solid non-skin cancers in RA patients during anti-TNF therapy, 446 RA patients with at least one diagnosis of cancer prior to the administration of TNFi were compared with 1278 controls with a history of equally recent cancer of the same type and stage [46]. Cancer recurrence incidence was 7\% in both RA patient cohorts. The study reassures clinicians that there is no increase in risk of cancer recurrence in RA patients with a history 
of cancer on TNFi compared with those on non-biologic treatment. However, that conclusion cannot be generalized to patients with a very recent cancer or poor prognosis and further investigations should be undertaken.

\section{Autoimmunity}

Autoimmune phenomena during biological therapy of rheumatic disease are related mainly to anti-TNF agents. For the last two decades of TNFi clinical use there have been reported a number of autoimmune diseases, principally drug-induced lupus and vasculitis-like events (VLES) and very rare events including interstitial lung disease, sarcoidosis, autoimmune uveitis, autoimmune hepatitis, and central and peripheral nervous system demyelination.

The characteristics of lupus and vasculitis like events are very diverse. Drug-induced lupus resolves within weeks to months after administration of a lupusinducing drug. Definition includes the presence of at least one SLE symptom (common symptoms: malaise, arthralgia, polyarthritis, myalgias, fever, serositis, rash) and antinuclear antibody (ANA) positivity or laboratory abnormalities including leukopenia, thrombocytopenia and increased erythrocyte sedimentation rate [47]. As regards VLEs, the types of the events range from limited cutaneous manifestation including urticarial, purpuric and ulcerating lesions to severe systemic involvement.

Treatment with TNFis may result in ANA induction and development of lupus-like events (LLES). An increasing number of studies have found that the incidence of ANAs in patients on TNFis is high. Bardazzi et al. [6] reported that $37 \%$ of 128 psoriatic patients treated with anti-TNF agents developed the antibodies and only one patient suffered from drug-induced lupus erythematosus. Out of three investigated drugs - adalimumab, etanercept and infliximab - the latter was the most implicated [6].

This observation is consistent with the results of other studies. In a group of 229 RA and ankylosing spondylitis patients treated with infliximab (159 patients) and etanercept (125 patients) induction of ANAs was observed only during infliximab therapy [48]. Only three patients were diagnosed with LLE. In a randomized phase III trial ANA seroconversion at low titers was observed in 54 of 342 (16\%) RA patients on infliximab and one patient suffered from LLE [49].

Immunogenicity of certolizumab pegol and golimumab is likely to be low. In a large randomized controlled trial only $2 \%$ of certolizumab-treated patients with Lesniowski-Crohn disease developed ANAs [50]. An analysis of golimumab safety performed by Kay et al. [51] showed that there is no statistically significant risk of autoimmu- nity during 3-year treatment in patients with RA, ankylosing spondylitis and psoriatic arthritis.

The studies suggest that there is a drug-specific difference in ANA induction and infliximab is the most potent. It may be due to the infliximab structure. It is a chimeric antibody and this may result in a greater immune response.

Although seroconversion to ANA positivity following anti-TNF agent usage is well known, the development of clinically evident autoimmune disease is rare. The former does not imply the latter, and routine ANA testing does not help clinicians to predict development of LLEs and VLEs [52]. There is a vast amount of literature on immune-mediated adverse effects in patients on anti-TNF treatment, mainly case reports, retrospective studies and spontaneous pharmacovigilance. In a French national retrospective study, it was found that LLEs include manifestations limited to skin symptoms (10/22 patients) and more severe systemic manifestations $(12 / 22)$. It was estimated that the incidence of LLES is very low $(0.19 \%$ with infliximab, $0.18 \%$ with etanercept, $0.10 \%$ with adalimumab) $[53,54]$. The risk of development of LLEs during certolizumab pegol or golimumab therapy is estimated to be even lower [51, 55], but it may be due to a shorter period of clinical use of the agents. Furthermore, it was found that switching from infliximab or adalimumab to certolizumab may cause resolution of LLEs [56].

Analysis regarding the VLE during anti-TNF therapy indicated that it is, similarly to LLEs, a rare event [57] and it is mainly limited to a skin manifestation, but visceral vasculitis with nervous system, renal and lung involvement was observed [58].

The retrospective studies provide information about the characteristic of events, but have a number of limitations, for example do not allow the incidence rate to be calculated $[53,57]$.

A prospective cohort study based on the British Society for Rheumatology Biologics Register-RA database (BSRBR-database), one of the largest biological registers, allowed the risk of AEs during biological treatment to be evaluated [59]. The most significant result of the study is that the absolute risk of the events during TNFi treatment is very small (10/10 000 patient-years for LLE, 15/10 000 patient-years for VLE). Moreover, the majority of events were limited to skin manifestations. The risk of the events was proved to be the highest for infliximab, which is in consistent with other studies. High disease activity (baseline DAS28 score and HAQ score) was proved to be associated with an increased risk of LLES and VLEs, while concomitant treatment with csDMARDs was proved to be associated with a lower risk. It has been calculated that there is no increased risk of LLEs and VLES 
in RA patients on anti-TNF agents ( $n=12$ 973) in comparison with a cohort receiving csDMARDs $(n=3673)$ and, therefore, the addition of TNFi to csDMARDs does not alter the risk of the events. To date, that is the sole prospective observational study that allows one to evaluate the actual risk of LLES and VLEs during anti-TNF therapy.

Among biological drugs approved for rheumatic diseases, beside anti-TNF agents, rituximab, tocilizumab and anakinra were linked to isolated cases of autoimmunity $[60,61]$. Further studies are needed to estimate the risk of autoimmune diseases in patients on bDMARDs.

TNF is well known as a proinflammatory cytokine. The fact that inhibition of TNF is associated with the onset of autoimmune diseases is surprising and indicates the complex role of TNF in the immune system. One possible explanation of this phenomena is that anti-TNF therapy disturbs the balance between regulatory $T$ cells (Tregs) and autoreactive effector T cells (Teffs). Autoreactive Teffs are present in healthy individuals and are persistently suppressed by Tregs [62]. TNF by signaling through TNF-receptor 2, which is highly expressed on Tregs, mediates signals promoting activation and proliferation [63]. Thus, a blockade of TNF by anti-TNF antibodies may result in downregulation of Treg activity and therefore in activation of autoreactive Teffs. Alternatively, anti-TNF antibodies may lead to autoimmunity by inducing apoptosis with release of nuclear autoantigens. Moreover, an increasing number of infections during anti-TNF therapy may induce polyclonal B-lymphocyte activation and production of antibodies and thus contribute to development of autoimmune-related diseases. Further investigations on the autoimmunity during TNFi treatment are required to elucidate the mechanism.

\section{Conclusions}

Adverse effects of bDMARDs are rare but clinically important. Safety data come from many sources. The most useful data for determining the risk of events are those from large national registries. To date, the risk of infections in RA patients on anti-TNF treatment has been shown to be significant.

Screening prior to initiating biological treatment of rheumatic diseases should include testing for tuberculosis, hepatitis B virus, hepatitis C virus and human immunodeficiency virus infections.

Regarding malignancies, there is no significant difference in the overall incidence of cancers in patients on anti-TNF treatment and the general population or patients on csDMARDs. There have been reports of an increased risk of hematologic malignancies and non-melanoma skin cancers in RA patients treated with TNFis and abatacept, respectively. Nevertheless, studies in a large cohort of patients are needed to evaluate this risk.

In patients on bDMARDs autoantibody induction is frequently observed, while development of autoimmune diseases is a rare event. Routine ANA testing in patients receiving biological therapy is not recommended. Autoimmunity during anti-TNF treatment seems to be related to pleiotropic functions of TNF in regulation of the immune system and further studies are needed to explain the pathogenesis of this phenomenon.

The authors declare no conflict of interest.

\section{References}

1. U.S. Food and Drug Administration. www.fda.gov (accessed 10 July 2018).

2. Salliot C, Dougados M, Gossec L. Risk of serious infections during rituximab, abatacept and anakinra treatments for rheumatoid arthritis: meta-analyses of randomised placebocontrolled trials. Ann Rheum Dis 2008; 68: 25-32.

3. Ribatti D. The concept of immune surveillance against tumors: The first theories. Oncotarget 2017; 8: 7175-7180.

4. Salavoura K, Kolialexi A, Tsangaris G, Mavrou A. Development of cancer in patients with primary immunodeficiencies. Anticancer Res 2008; 28: 1263-1269.

5. Choy E. Understanding the dynamics: pathways involved in the pathogenesis of rheumatoid arthritis. Rheumatology (Oxford) 2012; 51: v3-v11.

6. Bardazzi F, Odorici G, Virdi A, et al. Autoantibodies in psoriatic patients treated with anti-TNF- $\alpha$ therapy: Autoantibodies, clinical symptoms and treatment failure. J Dtsch Dermatol Ges 2014; 12: 401-406.

7. Galloway JB, Hyrich KL, Mercer LK, et al. Anti-TNF therapy is associated with an increased risk of serious infections in patients with rheumatoid arthritis especially in the first 6 months of treatment: updated results from the British Society for Rheumatology Biologics Register with special emphasis on risks in the elderly. Rheumatology (Oxford) 2011; 50: 124-131.

8. Askling J, Fored CM, Brandt L, et al. Time-dependent increase in risk of hospitalisation with infection among Swedish RA patients treated with TNF antagonists. Ann Rheum Dis 2007; 66: 1339-1344.

9. Singh JA, Cameron C, Noorbaloochi S, et al. Risk of serious infection in biological treatment of patients with rheumatoid arthritis: a systematic review and meta-analysis. Lancet 2015; 386: 258-265.

10. Cobo-lbáñez T, Descalzo MÁ, Loza-Santamaría E, et al. Serious infections in patients with rheumatoid arthritis and other immune-mediated connective tissue diseases exposed to anti-TNF or rituximab: data from the Spanish registry BIOBADASER 2.0. Rheumatol Int 2014; 34: 953-961.

11. Sakai R, Komano Y, Tanaka M, et al. Time-dependent increased risk for serious infection from continuous use of TNF antagonists during three years in rheumatoid arthritis patients. Arthritis Care Res 2012; 64: 1125-1134. 
12. Aaltonen KJ, Joensuu JT, Virkki L, et al. Rates of Serious Infections and Malignancies Among Patients with Rheumatoid Arthritis Receiving Either Tumor Necrosis Factor Inhibitor or Rituximab Therapy. J Rheumatol 2015; 42: 372-378.

13. Sakai R, Cho S-K, Nanki T, et al. Head-to-head comparison of the safety of tocilizumab and tumor necrosis factor inhibitors in rheumatoid arthritis patients (RA) in clinical practice: results from the registry of Japanese RA patients on biologics for longterm safety (REAL) registry. Arthritis Res Ther 2015; 17: 74.

14. Lampropoulos CE, Orfanos P, Bournia V-K, et al. Adverse events and infections in patients with rheumatoid arthritis treated with conventional drugs or biologic agents: a real world study. Clin Exp Rheumatol 2015; 33: 216-224.

15. van Dartel SAA, Fransen J, Kievit W, et al. Difference in the risk of serious infections in patients with rheumatoid arthritis treated with adalimumab, infliximab and etanercept: results from the Dutch Rheumatoid Arthritis Monitoring (DREAM) registry. Ann Rheum Dis 2013; 72: 895-900.

16. Richter A, Listing J, Schneider M, et al. Impact of treatment with biologic DMARDs on the risk of sepsis or mortality after serious infection in patients with rheumatoid arthritis. Ann Rheum Dis 2016; 75: 1667-1673.

17. Parameswaran N, Patial S. Tumor necrosis factor- $\alpha$ signaling in macrophages. Crit Rev Eukaryot Gene Expr 2010; 20: 87-103.

18. Józefowski S, Sobota A, Hamasur B, Kwiatkowska K. Mycobacterium tuberculosis lipoarabinomannan enhances LPS-induced TNF- $\alpha$ production and inhibits NO secretion by engaging scavenger receptors. Microb Pathog 2011; 50: 350-359.

19. Quesniaux VFJ, Jacobs M, Allie N, et al. TNF in Host Resistance to Tuberculosis Infection. Curr Dir Autoimmun 2010; 11: 157-179.

20. Botha T, Ryffel B. Reactivation of latent tuberculosis infection in TNF-deficient mice. J Immunol 2003; 171: 3110-3118.

21. Arkema EV, Jonsson J, Baecklund E, et al. Are patients with rheumatoid arthritis still at an increased risk of tuberculosis and what is the role of biological treatments? Ann Rheum Dis 2015; 74: 1212-1217.

22. Jick SS, Lieberman ES, Rahman MU, Choi HK. Glucocorticoid use, other associated factors, and the risk of tuberculosis. Arthritis Rheum 2006; 55: 19-26.

23. Miller EA, Ernst JD. Anti-TNF immunotherapy and tuberculosis reactivation: another mechanism revealed. J Clin Invest 2009 119: 1079-1082.

24. Chen DY, Shen GH, Chen YM, et al. Biphasic emergence of active tuberculosis in rheumatoid arthritis patients receiving TNF $\alpha$ inhibitors: the utility of IFN $\gamma$ assay. Ann Rheum Dis 2012; 71: 231-237.

25. Ramos JM, Robledano C, Masiá M, et al. Contribution of Interferon gamma release assays testing to the diagnosis of latent tuberculosis infection in HIV-infected patients: A comparison of QuantiFERON-TB Gold In Tube, T-SPOT.TB and tuberculin skin test. BMC Infect Dis 2012; 12: 169.

26. Singh JA, Saag KG, Bridges SL, et al. 2015 American College of Rheumatology Guideline for the Treatment of Rheumatoid Arthritis: ACR RA Treatment Recommendations. Arthritis Care Res (Hoboken) 2016; 68: 1-25.

27. Yawn BP, Saddier P, Wollan PC, et al. A population-based study of the incidence and complication rates of herpes zoster before zoster vaccine introduction. Mayo Clin Proc 2007; 82: 1341-1349.
28. Pappas DA, Hooper MM, Kremer JM, et al. Herpes Zoster Reactivation in Patients With Rheumatoid Arthritis: Analysis of Disease Characteristics and Disease-Modifying Antirheumatic Drugs: Risk Conferred by DMARDs on HZ Reactivation in RA. Arthritis Care Res 2015; 67: 1671-1678.

29. Marra F, Lo E, Kalashnikov V, Richardson K. Risk of Herpes Zoster in Individuals on Biologics, Disease-Modifying Antirheumatic Drugs, and/or Corticosteroids for Autoimmune Diseases: A Systematic Review and Meta-Analysis. Open Forum Infect Dis 2016; 3: ofw205.

30. Smitten AL, Choi HK, Hochberg MC, et al. The risk of herpes zoster in patients with rheumatoid arthritis in the United States and the United Kingdom. Arthritis Rheum 2007; 57 : 1431-1438

31. Tan CS, Koralnik IJ. Beyond progressive multifocal leukoencephalopathy: expanded pathogenesis of JC virus infection in the central nervous system. Lancet Neurol 2010; 9: 425-437.

32. Berger JR, Malik V, Lacey S, et al. Progressive multifocal leukoencephalopathy in rituximab-treated rheumatic diseases: a rare event. J Neurovirol 2018; 24: 323-331.

33. Stearns-Kurosawa DJ, Osuchowski MF, Valentine C, et al. The Pathogenesis of Sepsis. Annu Rev Pathol 2011; 6: 19-48.

34. Lv S, Han M, Yi R, et al. Anti-TNF- $\alpha$ therapy for patients with sepsis: a systematic meta-analysis. Int J Clin Pract 2014; 68: 520-528.

35. Fisher CJ Jr, Dhainaut JF, Opal SM, et al. Recombinant human interleukin 1 receptor antagonist in the treatment of patients with sepsis syndrome. Results from a randomized, double-blind, placebo-controlled trial. Phase III rhIL-1ra Sepsis Syndrome Study Group. JAMA 1994; 271: 1836-1843.

36. Tracey KJ, Fong Y, Hesse DG, et al. Anti-cachectin/TNF monoclonal antibodies prevent septic shock during lethal bacteraemia. Nature 1987; 330: 662-664.

37. Bongartz T, Sutton AJ, Sweeting MJ, et al. Anti-TNF Antibody Therapy in Rheumatoid Arthritis and the Risk of Serious Infections and Malignancies: Systematic Review and Meta-analysis of Rare Harmful Effects in Randomized Controlled Trials. JAMA 2006; 295: 2275

38. Carmona L, Abasolo L, Descalzo MA, et al. Cancer in Patients with Rheumatic Diseases Exposed to TNF Antagonists. Semin Arthritis Rheum 2011; 41: 71-80.

39. Strangfeld A, Hierse F, Rau R, et al. Risk of incident or recurrent malignancies among patients with rheumatoid arthritis exposed to biologic therapy in the German biologics register RABBIT. Arthritis Res Ther 2010; 12: R5.

40. Mercer LK, Lunt M, Low ALS, et al. Risk of solid cancer in patients exposed to anti-tumour necrosis factor therapy: results from the British Society for Rheumatology Biologics Register for Rheumatoid Arthritis. Ann Rheum Dis 2015; 74: 1087-1093.

41. Berghen N, Teuwen L-A, Westhovens R, Verschueren P. Malignancies and anti-TNF therapy in rheumatoid arthritis: a single-center observational cohort study. Clin Rheumatol 2015; 34: 1687-1695.

42. Mercer LK, Galloway JB, Lunt M, et al. Risk of lymphoma in patients exposed to antitumour necrosis factor therapy: results from the British Society for Rheumatology Biologics Register for Rheumatoid Arthritis. Ann Rheum Dis 2017; 76: 497-503.

43. Wadström H, Frisell T, Sparén P, Askling J. Do RA or TNF inhibitors increase the risk of cervical neoplasia or of recurrence 
of previous neoplasia? A nationwide study from Sweden. Ann Rheum Dis 2016; 75: 1272-1278.

44. Wadström H, Frisell T, Askling J. Malignant Neoplasms in Patients With Rheumatoid Arthritis Treated With Tumor Necrosis Factor Inhibitors, Tocilizumab, Abatacept, or Rituximab in Clinical Practice: A Nationwide Cohort Study From Sweden. JAMA Intern Med 2017; 177: 1605.

45. Alten R, Kaine J, Keystone E, et al. Long-Term Safety of Subcutaneous Abatacept in Rheumatoid Arthritis: Integrated Analysis of Clinical Trial Data Representing More Than Four Years of Treatment: Long-Term Safety of Subcutaneous Abatacept. Arthritis Rheumatol 2014; 66: 1987-1997.

46. Raaschou P, Söderling J, Askling J, et al. OP0308 Tnf inhibitor treatment and risk of cancer recurrence in patients with rheumatoid arthritis: a nationwide cohort study from sweden. Ann Rheum Dis 2017; 76: 183.

47. Puszczewicz M (ed.). Rheumatology. Medical Tribune Poland, Warsaw 2010

48. Gonnet-Gracia C, Barnetche T, Richez C, et al. Anti-nuclear antibodies, anti-DNA and C4 complement evolution in rheumatoid arthritis and ankylosing spondylitis treated with TNFalpha blockers. Clin Exp Rheumatol 2008; 26: 401-407.

49. Maini R, St Clair EW, Breedveld F, et al. Infliximab (chimeric anti-tumour necrosis factor alpha monoclonal antibody) versus placebo in rheumatoid arthritis patients receiving concomitant methotrexate: a randomised phase III trial. ATTRACT Study Group. Lancet 1999; 354: 1932-1939.

50. Sandborn WJ, Feagan BG, Stoinov S, et al. Certolizumab Pegol for the Treatment of Crohn's Disease. N Engl J Med 2007; 357 228-238.

51. Kay J, Fleischmann R, Keystone E, et al. Golimumab 3-year safety update: an analysis of pooled data from the long-term extensions of randomised, double-blind, placebo-controlled trials conducted in patients with rheumatoid arthritis, psoriatic arthritis or ankylosing spondylitis. Ann Rheum Dis 2015; 74: 538-546.

52. Takase K, Horton SC, Ganesha A, et al. What is the utility of routine ANA testing in predicting development of biological DMARD-induced lupus and vasculitis in patients with rheumatoid arthritis? Data from a single-centre cohort. Ann Rheum Dis 2014; 73: 1695-1699.
53. De Bandt M, Sibilia J, Le Loët X, et al. Systemic lupus erythematosus induced by anti-tumour necrosis factor alpha therapy: a French national survey. Arthritis Res Ther 2005; 7: R545-551.

54. Schiff MH. Safety analyses of adalimumab (HUMIRA) in global clinical trials and US postmarketing surveillance of patients with rheumatoid arthritis. Ann Rheum Dis 2005; 65: 889-894.

55. Bykerk VP, Cush J, Winthrop K, et al. Update on the safety profile of certolizumab pegol in rheumatoid arthritis: an integrated analysis from clinical trials. Ann Rheum Dis 2015; 74: 96-103.

56. Verma HD, Scherl EJ, Jacob VE, Bosworth BP. Anti-nuclear antibody positivity and the use of certolizumab in inflammatory bowel disease patients who have had arthralgias or lupus-like reactions from infliximab or adalimumab: Certolizumab and lupus-like reactions. J Dig Dis 2011; 12: 379-383.

57. Saint Marcoux B, De Bandt M. Vasculitides induced by TNFalpha antagonists: a study in 39 patients in France. Joint Bone Spine 2006; 73: 710-713.

58. Ramos-Casals M, Brito-Zerón P, Muñoz S, et al. Autoimmune Diseases Induced by TNF-Targeted Therapies: Analysis of 233 Cases. Medicine (Baltimore) 2007; 86: 242-251.

59. Jani M, Dixon WG, Kersley-Fleet L, et al. Drug-specific risk and characteristics of lupus and vasculitis-like events in patients with rheumatoid arthritis treated with TNFi: results from BSRBR-RA. RMD Open 2017; 3: e000314.

60. Kim MJ, Kim HO, Kim HY, Park YM. Rituximab-induced vasculitis: A case report and review of the medical published work. J Dermatol 2009; 36: 284-287.

61. Sakaue S, Sumitomo S, Kubo K, et al. Tocilizumab-induced leucocytoclastic vasculitis in a patient with rheumatoid arthritis. Rheumatology (Oxford) 2014; 53: 1529-1530.

62. Danke NA, Koelle DM, Yee C, et al. Autoreactive T Cells in Healthy Individuals. J Immunol 2004; 172: 5967-5972.

63. Chen X, Wu X, Zhou Q, et al. TNFR2 Is Critical for the Stabilization of the CD4+Foxp3+ Regulatory T Cell Phenotype in the Inflammatory Environment. J Immunol 2013; 190: 1076-1084. 